\title{
Diseño de seguros ganaderos paramétricos satelitales de tipo catastrófico: implementación operacional en México
}

\section{Mexican experience on the design of catastrophic satellital parametric livestock insurances: operational implementation}

\author{
Fernando Paz ${ }^{1 \ddagger}$, Martín Bolaños ${ }^{1}$, Fermín Pascual ${ }^{1}$, Jesús Escamilla ${ }^{2}$, \\ Mario Cuesta ${ }^{2}$ y José Inés Zúñiga ${ }^{2}$
}

\footnotetext{
${ }^{1}$ Colegio de Postgraduados, Campus Montecillo. Carretera México-Texcoco km. 36.5. 56230 Montecillo, Estado de México, México.

Autor responsable (ferpazpel@gmail.com)

${ }^{2}$ AGROASEMEX, S.A. Avenida Constituyentes 124 Poniente, Colonia El Carrizal. 76030 Santiago de Querétaro, Querétaro, México.
}

\section{RESUMEN}

La implementación operacional de esquemas de gestión de riesgos climáticos, principalmente seguros, es una opción para mitigar los efectos del cambio climático y hacer un uso eficiente de los recursos financieros a escala regional y de país. En el presente trabajo se presenta la experiencia operativa en la implementación de seguros en México, misma que se fundamenta en el desarrollo conceptual-teórico de su marco de implementación. El diseño de seguros ganaderos paramétricos satelitales se basa en la relación $\mathrm{Bm}=\mathrm{kIV}$, donde $\mathrm{Bm}$ es la biomasa aérea total, IV es un índice espectral de la vegetación y $\mathrm{k}$ es una constante empírica. Para lograr esta relación, se utiliza una relación lineal entre el índice espectral NDVIcp y la Bm, la cual se transforma en el índice IVCP (en función de 1/NDVIcp). La ventaja de usar el índice transformado 1/NDVIcp es que tiene patrones lineales en el tiempo, los cuales se pueden usar para su parametrización en términos operacionales. Este desarrollo se discute mediante el uso de un enfoque algorítmico que considera la parametrización de curvas de crecimiento de la vegetación, índice 1/NDVIcp, para el sensor AVHRR. Se presenta, también, la experiencia operacional de la implementación en México y se incluyen las campañas de validación. De la experiencia operativa se concluye que, en un ciclo completo, aún cuando el esquema que se desarrolló reduce los riesgos de base que se asocian al uso de periodos cortos del

Cita recomendada:

Paz, F., M. Bolaños, F. Pascual, J. Escamilla, M. Cuesta y J. Inés Zúñiga. 2018. Diseño de seguros ganaderos paramétricos satelitales de tipo catastrófico: implementación operacional en México. Terra Latinoamericana 36: 141-152. DOI: https://doi.org/10.28940/terra.v36i2.231 seguro, permanece el problema de ciclos del crecimiento desfasados, lo que constituye una restricción inherente al considerar una fecha inicial y final de vigencia del seguro paramétrico. En esta perspectiva se proponen modificaciones para desarrollos futuros.

Palabras clave: NDVIcp, IVCP, modelo paramétrico, sensor AVHRR, transformaciones lineales.

\section{SUMMARY}

The operational implementation of schemes for climate risk management, primarily insurance products, is an option to mitigate the effects of climate change and make efficient use of financial resources at regional and country levels. In this study the operational experience in implementing parametric insurance products in Mexico, based on the conceptual-theoretical development of the implementation framework, is presented. Parametric design of livestock insurance satellite products is based on the relation $\mathrm{Bm}=\mathrm{kVI}$, where $\mathrm{Bm}$ is the total aerial biomass, VI is a spectral vegetation index and $\mathrm{k}$ is an empirical constant. To achieve this relationship, a linear relationship between the spectral index NDVIcp and the Bm, which becomes the index IVCP (1/NDVIcp) is used. The advantage of using the transformed 1/NDVIcp index is having linear patterns over time, which can be used for parameterization in operational terms. This development is discussed by using an algorithmic

Recibido: febrero de 2017. Aceptado: marzo de 2018 Terra Latinoamericana 36: 141-152. 
approach that considers the parameterization of vegetation growth curves, index 1/NDVIcp, for the AVHRR sensor. It also presents the operational experience of implementation in Mexico and validation campaigns are included. From operational experience, it is concluded that, in a complete cycle, even if the scheme developed reduces the basic risks associated with the use of short periods of insurance, the problem of growth cycle lags remains, constituting an inherent restriction when considering the beginning and end date of parametric insurance cover. Recommendations are given to improve the insurance products.

Index words: NDVIcp, IVCP, parametric model, AVHRR sensor, linear transformations.

\section{INTRODUCCIÓN}

El riesgo climático, particularmente los eventos extremos como las sequías, heladas e inundaciones, es un factor que requiere modelarse en términos técnicos para poder desarrollar esquemas de gestión de riesgos, tanto de retención y manejo, como de transferencia (AGROASEMEX, 2005; Arias y Covarrubias, 2006). La tecnología de los sensores remotos permite la generación de estadísticas históricas a nivel de un píxel de una imagen satelital, para valorar el riesgo de eventos extremos que inciden en la operación de las actividades ganaderas.

Los sensores remotos utilizan el alto contraste entre la banda fotosintética del rojo (R) y la no fotosintética del infrarrojo cercano (IRC), para caracterizar el estado de la vegetación con relación a otros objetos terrestres (Tucker, 1979). Los índices espectrales de la vegetación (IV) usan las bandas del $\mathrm{R}$ y del IRC para obtener relaciones con las variables biofísicas (biomasa o Bm, índice de área foliar o IAF y cobertura aérea o $\mathrm{COB}$ ) asociadas con la vegetación (Verstraete y Pinty, 1996; Paz et al., 2007). Los índices de vegetación, como el NDVIcp, se diseñaron para minimizar el efecto del suelo que subyace a ésta (Paz et al., 2007), los efectos atmosféricos (Paz et al., 2008) y, la geometría sol-sensor (Bolaños et al., 2007). Bajo estas consideraciones, los IV se relacionan fuertemente con la vegetación y no con los efectos que conlleva la tecnología de los sensores remotos y el procesamiento e integración de la información espectral.
El uso de IV para el diseño de seguros ganaderos paramétricos satelitales del tipo catastrófico, se llegó a plantear como un esquema viable para su operación rutinaria (AGROASEMEX, 2006; Paz et al., 2006). En este trabajo se presentan las consideraciones de tipo operacional que se requieren para la implementación de seguros ganaderos paramétricos satelitales.

\section{MATERIALES Y MÉTODOS}

Como primera etapa se llevó a cabo el análisis y adecuación del marco conceptual-teórico en el que se basa la implementación de los seguros ganaderos paramétricos satelitales, para posteriormente establecer la parte operacional.

\section{Marco Conceptual Teórico}

\section{Índice NDVIcp y su Relación con la Biomasa}

En Paz et al. (2018) se presentan, a nivel detallado, los requerimientos esenciales de un seguro paramétrico, mismos que pueden resumirse en:

$$
B m=k I V
$$

donde $\mathrm{k}$ es una constante empírica, que depende de cada tipo de vegetación y entorno físico-ecológico.

El índice NDVIcp (Paz et al., 2007) se diseñó para contar con una relación lineal con el IAF en la etapa vegetativa (hasta un IAF aproximado entre 2 y 3 ). Si se considera que el IAF tiene una relación lineal con la Bm (Goudriaan y van Laar, 1994), se pueden hacer estimaciones robustas de la biomasa. Cuando la vegetación pasa cierto umbral de estrés, la relación entre el IAF y la Bm se relaja (las plantas incrementan sus reservas de fotosintatos en la parte subterránea), lo que modifica la relación entre la biomasa aérea y la producción foliar (Stockle et al., 2003), aún cuando la relación permanece aproximadamente lineal.

Con datos provenientes de un experimento con algodón (Huete et al., 1985), mismo que se discute en Paz et al. (2007), se elaboró la Figura 1a, que muestra la relación entre el índice NDVIcp con la biomasa aérea seca (etapa vegetativa y reproductiva), en la que destaca, una relación no lineal general. 

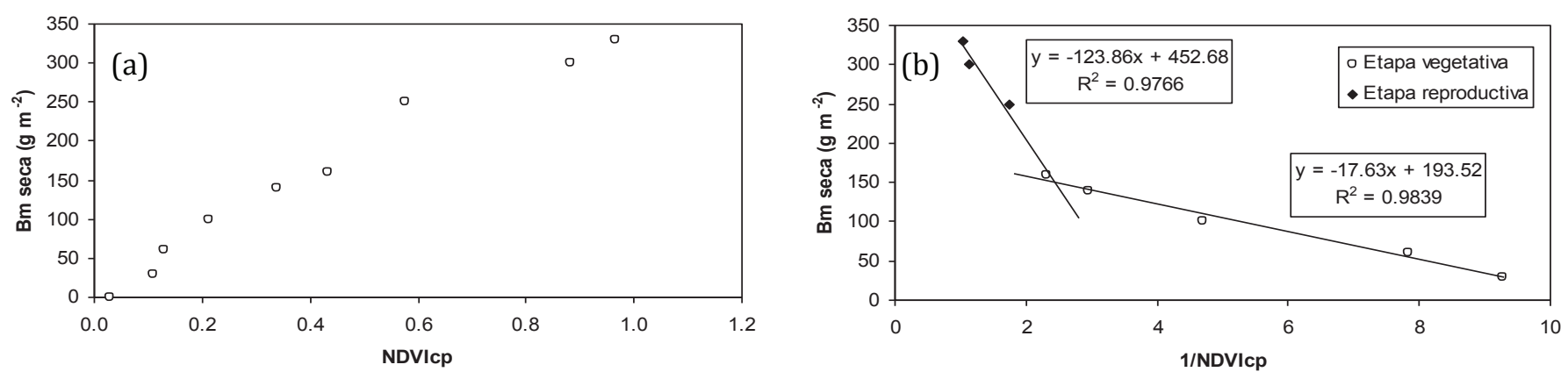

Figura 1. (a) Relación entre la Bm (biomasa) seca y el NDVIcp y (b) relación entre la Bm seca y 1/NDVIcp.

Para hacer lineal la relación entre el NDVIcp y Bm (biomasa seca), se realizó una transformación simple del índice (1/NDVIcp), lo que da como resultado un patrón bilineal, lineal para la etapa vegetativa, Figura $1 \mathrm{~b}$. Si se considera que el seguro ganadero paramétrico satelital se diseñó para pastizales naturales/inducidos y matorrales (Paz et al., 2018), la aproximación de la etapa vegetativa se considera suficiente para fines operacionales.

\section{Curva de Crecimiento de la Vegetación Usando el Índice NDVIcp}

Para explicar el patrón de crecimiento del NDVIcp, se usaron datos experimentales de un pastizal natural (Bouteloua spp.) en la cuenca de Walnut Gulch en Arizona, Estados Unidos, cuya descripción detallada se encuentra en Huete et al. (1992), Chehbouni et al. (1994) y Qi et al. (1994). A partir de dichos datos, Bolaños et al. (2007) desarrollaron un modelo de la geometría sol-sensor de las reflectancias, del que se desprende la Figura 2, que muestra los patrones temporales de 1 / NDVIcp del pasto y destaca que las etapas vegetativas y de senescencia pueden aproximarse por un modelo lineal.

\section{Modelo Temporal General Propuesto para 1/NDVIcp}

Con base en los patrones expuestos en la Figura 2, la Figura 3 muestra el modelo propuesto para la evolución temporal $(\mathrm{X}=$ día juliano) de 1/NDVIcp $(=\mathrm{Y})$.

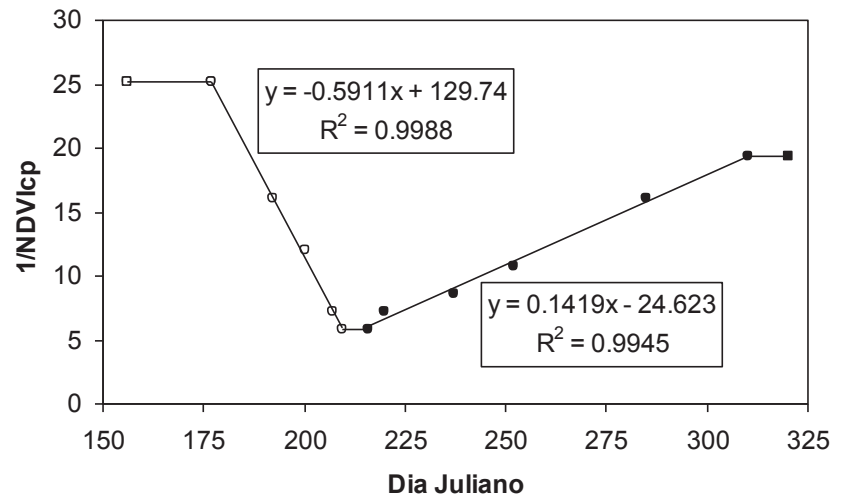

Figura 2. Patrones temporales de 1/NDVIcp para un pasto. Los puntos cuadrados fueron puestos en forma artificial para las mesetas.

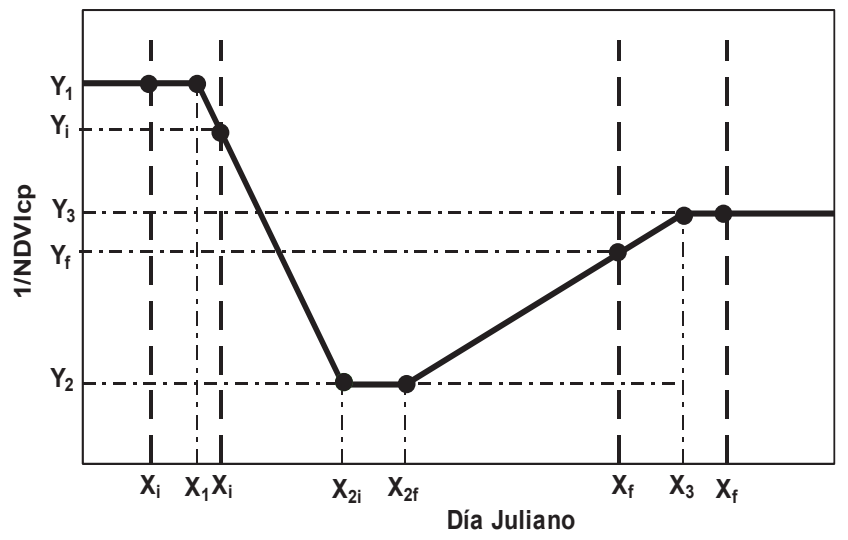

Figura 3. Modelo propuesto para la evolución temporal de 1/NDVIcp. 
Como se observa en la Figura 3, al inicio de la etapa vegetativa se presenta una meseta inicial (suelo desnudo o vegetación perenne). La etapa vegetativa inicia en $\left(X_{1}, Y_{1}\right)$ y termina en $\left(X_{2 i}, Y_{2}\right)$. Posteriormente hay una meseta intermedia entre $\left(X_{2 i}, Y_{2}\right)$ y $\left(X_{2 f}, Y_{2}\right)$ $y$, al final, una etapa de senescencia entre $\left(X_{2 f}, Y_{2}\right)$ $\mathrm{y}\left(\mathrm{X}_{3}, \mathrm{Y}_{3}\right)$. Al término de la senescencia se presenta una meseta final que corresponde a la dormancia o vegetación perenne. Con base en lo anterior, el ciclo del seguro se define por un inicio $X_{\mathrm{i}} \mathrm{y}$ un final $\mathrm{X}_{\mathrm{f}}$, $\sin$ que su presencia se limite al ciclo de crecimiento de la vegetación, es decir, que pueden ubicarse fuera de éste.

Las dos etapas de crecimiento de la vegetación, si se utiliza1/NDVIcp, pueden estimarse a partir de:

$$
\begin{aligned}
& \left(\frac{1}{N D V I c p}\right)=A_{1}+B_{1} t, \text { para la etapa vegetativa } \\
& \left(\frac{1}{N D V I c p}\right)=A_{2}+B_{2} t, \text { para la etapa de senescencia }
\end{aligned}
$$

Dado que la relación entre 1/NDVIcp y la Bm es lineal con una constante aditiva (Figura 1b), es necesario hacer una transformación para obtener una relación similar a la que se muestra en la Ecuación (1):

$I V C P=\left(\frac{1}{N D V I c p}\right)_{\min }-\left(\frac{1}{N D V I c p}\right)$

Así, el nuevo índice espectral IVCP (Índice de Vegetación del Colegio de Postgraduados) permite obtener una relación similar a la de la ecuación (1), ya que la constante aditiva entre 1/NDVIcp y Bm se filtra. El valor de (1/NDVIcp) ${ }_{\text {min }}$ se obtiene de la evolución temporal del índice 1/NDVIcp, tal como se describe a continuación.

$\mathrm{Si}$ se considera a las etapas vegetativa y de senescencia en forma independiente, se puede usar $(1 / \mathrm{NDVIcp})_{\min }=\mathrm{Y}_{1}$ en la etapa vegetativa $\mathrm{y}$, $(1 / \text { NDVIcp })_{\min }=Y_{3}$ en la de senescencia. La Figura 4 muestra el modelo del IVCP para esta estrategia de parametrización de (1/ NDVIcp) $)_{\text {min }}$.

\section{Definición del Índice del Seguro}

Aún cuando para la estimación de la capacidad de carga sólo se requiere conocer el pico (máxima producción forrajera) de la curva de crecimiento de la biomasa, la limitación del concepto estriba en que no considera la parte cualitativa (nutrimental) del forraje.

De acuerdo con Emmick (2000), la curva de crecimiento de un pasto consta de tres fases, sin considerar completamente la curva de la etapa de senescencia: a) En la etapa inicial del crecimiento, fase $I$, la vegetación consiste en hojas jóvenes o inmaduras, con alto contenido nutrimental, la calidad es alta, la producción (cantidad de biomasa) es escasa $\mathrm{y}$, si la vegetación está dispersa, la disponibilidad de alimento para el ganado es pequeña. Si se considera que el ganado bovino es fuertemente selectivo, el esfuerzo de ingerir esta pequeña dotación de alimento es mayor que la ganancia de la actividad. b) En la etapa de madurez, fase II, la vegetación está compuesta de hojas maduras y tallos. Al inicio de esta fase la mayoría de las hojas son altamente nutritivas, pero al final se reduce rápidamente su valor nutrimental. Es en esta etapa en la que se presenta la máxima producción de biomasa, por lo que define un intervalo óptimo de forrajeo para el ganado. c) En la fase III, de post madurez, la vegetación está compuesta principalmente de tallos, con hojas post maduras, por lo que su valor nutrimental declina rápidamente. El control de esta fase corresponde al valor nutrimental de la vegetación.

A partir de la discusión de la calidad forrajera de la vegetación, resulta claro que es importante contar con una medición de biomasa verde (consideración nutrimental) y no de biomasa seca, de tal manera que

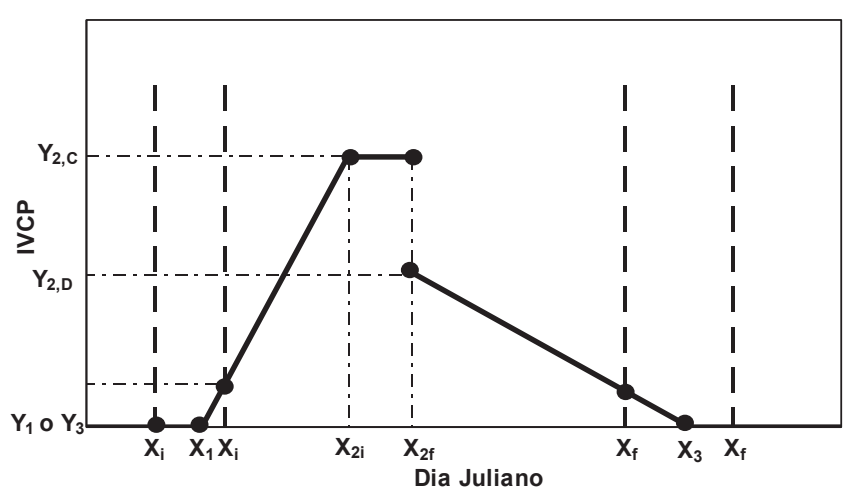

Figura 4. Patrón temporal del IVCP parametrizado en forma independiente para la etapa vegetativa y de senescencia. La letra $\mathrm{C}$ en los valores de $\mathrm{Y}$ (IVCP) se refiere a la etapa de crecimiento (vegetativa) y la letra $D$ a la de decrecimiento (senescencia). 
la curva de crecimiento se asemeje más a la del IAF, condiciones para las que el NDVIcp (e IVCP) resulta más confiable (Paz et al., 2007).

Como el índice de vegetación espectral representa una variable acumulada, en forma directa aporta la operación de integración (área bajo la curva de la tasa de crecimiento diario). En especial, un índice de vegetación espectral es una medida de la cantidad de hojas y tallos (alimento), así como de su calidad (pigmentos de las hojas y tallos). En este sentido, un IV constituye una medida directa del alimento disponible para el ganado, además de tener la característica de ser una medición promedio del área de un píxel (lo que no se puede realizar, en términos económicos, con las mediciones directas de biomasa).

Si se considera que el diseño del seguro ganadero paramétrico satelital sólo contempla el crecimiento anual de la vegetación (porque la vegetación perenne se filtra en el IVCP), es posible utilizar un índice de seguro (IS) que considere la cantidad y calidad del forraje.

Cuando sólo se incluye la cantidad de vegetación disponible, el IS se define por:

$I S=\left(Y_{1}-Y_{2}\right)+\left(Y_{3}-Y_{2}\right)=Y_{2, C}+Y_{2, D}$

que representa el crecimiento anual sólo de las etapas vegetativa y de senescencia, ya que durante la intermedia no hay crecimiento.

Para considerar la calidad de la biomasa en las etapas vegetativa y de senescencia, se requiere incorporar un esquema de ponderación en función de la digestibilidad o D (porcentaje del forraje que ingiere y metaboliza el animal), de tal manera que refleje la parte nutrimental del alimento, además de la cantidad (Holechek et al., 1989; USDA, 1999). Los factores críticos para el aprovechamiento del alimento que se ingiere son la cantidad de biomasa y sus propiedades nutrimentales: digestibilidad y proteína (USDA, 1999). Los valores de digestibilidad durante el crecimiento activo del forraje (vegetación) son generalmente superiores al 50\% e inferiores durante el periodo de dormancia (Holechek et al., 1989).

Para la ponderación de la biomasa en términos de su digestibilidad, se utilizó el modelo del USDA (Arnold et al., 1995) que considera el producto de la cantidad de biomasa que se ingiere, por su digestibilidad como fracción:

$$
B m D=(D r D m x) B m v+(1-D r) D m n B m m
$$

donde: Dmx es la digestibilidad máxima, Dmn la digestibilidad mínima, Bmv la biomasa aérea viva en pie, Bmm la biomasa aérea muerta en pie y Dr un factor que considera las proporciones entre biomasa viva $\mathrm{y}$ muerta:

$$
\begin{aligned}
& D r=1-\exp (-5 D l) \\
& D l=\frac{B m v}{B m m}
\end{aligned}
$$

En las relaciones (6) la razón Dl varía entre 0.1 (Dr $=0.39)$ y $1.0(\mathrm{Dr}=0.99)$. En un plan conservador, es posible usar la condición $\mathrm{Bmv}=0$ para el punto de la meseta final de la vegetación (dormancia), que da un valor de $\mathrm{Dr}=0.4$. Para la etapa vegetativa (creciente) y la meseta intermedia se puede usar un valor de Bmv $=1$, por lo que $\mathrm{Dr}=1.0$. De esta forma, la relación (5), lineal, se expresa como:

$$
B m D=D m x B m v+0.6 D m n B m m
$$

Si se usan valores de Dmx $=1.0$ y Dmn $=0.75$, en forma conservadora, la relación (7) se puede presentar como:

$B m D=1 x B m v+0.45 x B m m$

De esta manera, $\mathrm{BmD}$ corresponde a una biomasa con ajustes por calidad y el índice espectral de la vegetación IVCP ya sólo requiere de ponderación:

$I S=\left(Y_{1}-Y_{2}\right) x 1+\left(Y_{3}-Y_{2}\right) x F D$

El factor de digestibilidad FD (relativo) sólo se aplica a la etapa de senescencia (vale 1.0 en la etapa de crecimiento y de meseta) y parte de un valor relativo de 1.0 (digestibilidad máxima) al inicio de la senescencia, hasta un valor de $0.45(0.60 \times 0.75)$ en la meseta final o etapa de dormancia de la vegetación, con una variación lineal en el tiempo. La Figura 5 muestra el esquema de ponderación mediante el uso del modelo de crecimiento de la vegetación.

La estrategia de ponderación que se implementó para la estimación del índice del seguro (IS), además de considerar en forma explícita el valor nutrimental de la biomasa, también reduce los efectos de problemas que 


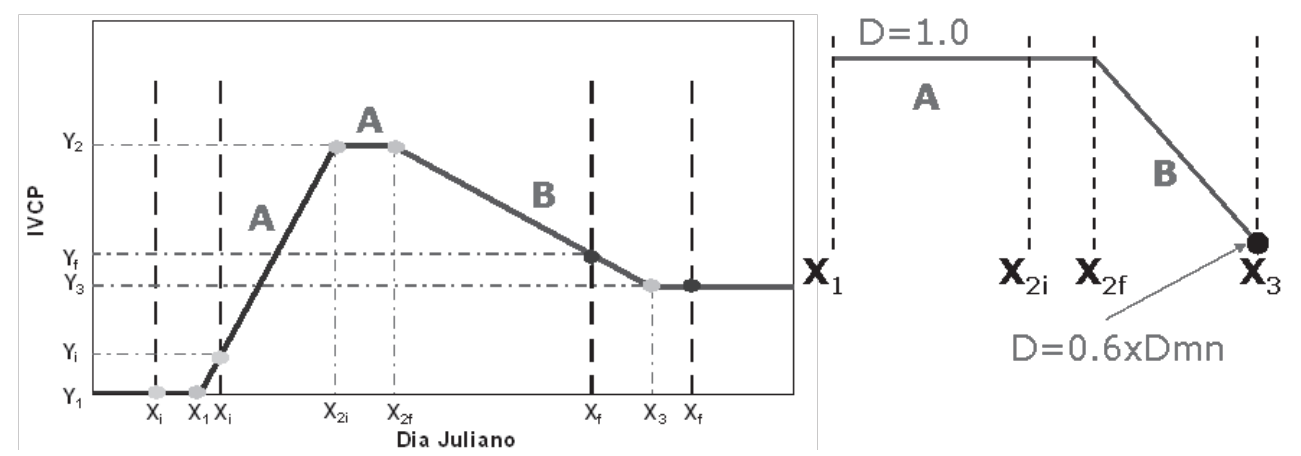

Figura 5. Esquema de ponderación del índice IVCP por la digestibilidad de la biomasa.

conllevan eventos extremos en la etapa de senescencia, como las heladas tempranas. El esquema de ponderación propuesto, por lo tanto, es suficientemente simple y con fundamentos biofísicos sólidos, para implementarse en forma operacional.

\section{Implementación Operacional del Seguro Paramétrico}

Para contar con un registro histórico de curvas de crecimiento anuales a nivel de píxel e integrarlas en regiones para valorar el riesgo asociado a eventos climáticos extremos, se utilizaron imágenes satelitales del sensor AVHRR (Kidwell, 1998; Goodrum et al., 2001), disponibles para México desde 1986 (cobertura nacional).

El procesamiento de las imágenes ( $\mathrm{Paz}$ et al., 2011) se realizó en seis etapas: i) Se referenciaron geográficamente con un modelo orbital para su reproyección en coordenadas cónicas conforme de Lambert y se integraron en una malla nacional con celdas de $1 \mathrm{~km} \times 1 \mathrm{~km}$, con el método del vecino más cercano. El error de referenciación fue de 1 a 3 píxeles, lo que no repercute en la implementación del seguro a nivel de municipios de México. ii) Para evitar el procesamiento de píxeles que se relacionan con nubes y sombras, se utilizó una adaptación del algoritmo de discriminación de nubes CLAVR-1 de la NASA (Stowe et al., 1999). Sólo se procesaron los píxeles correspondientes a la mezcla suelo-vegetación por día juliano y píxel individual. iii) Para cada píxel se estimó el índice NDVIcp (Paz et al., 2007), después de proyectar las reflectancias a nadir con el modelo de geometría sol-sensor de Bolaños et al. (2007). Los parámetros empíricos del NDVIcp se optimizaron con el fin de reducir los efectos atmosféricos (Paz et al., 2008). iv) El vector de índices NDVIcp (días julianos) de cada píxel de la malla nacional se usó para estimar los parámetros de la curva de crecimiento de la vegetación (Figura 3), para lo cual se utilizó un algoritmo de reconocimiento de patrones lineales por etapa de crecimiento, dado que los índices NDVIcp contenían efectos residuales de las correcciones que se realizaron (paso iii). La Figura 6 muestra un ejemplo del resultado de este proceso para un píxel. Posteriormente, se almacenaron los parámetros resultado del ajuste de la curva de crecimiento de 1/NDVIcp: $\mathrm{Y}_{1}, \mathrm{Y}_{2}, \mathrm{Y}_{2 \text { int }}{ }^{-}$ intersección de la curva creciente y decreciente -, $\mathrm{Y}_{3}, \mathrm{~A}_{1}, \mathrm{~B}_{1}, \mathrm{~A}_{2}, \mathrm{~B}_{2}$, donde los parámetros $\mathrm{X}_{1}, \mathrm{X}_{2 \mathrm{i}}, \mathrm{X}_{2 \mathrm{f}}$ y $\mathrm{X}_{3}$ se obtienen de los parámetros anteriores. v) Con los parámetros de las curvas de crecimiento anual de cada píxel y mediante el uso de promedios ponderados para su integración a escala de municipios (Paz et al., 2018), se estimó el índice del seguro, relación (9), lo que permitió su tarificación (valoración del riesgo y costo de la prima del seguro) para el seguro ganadero

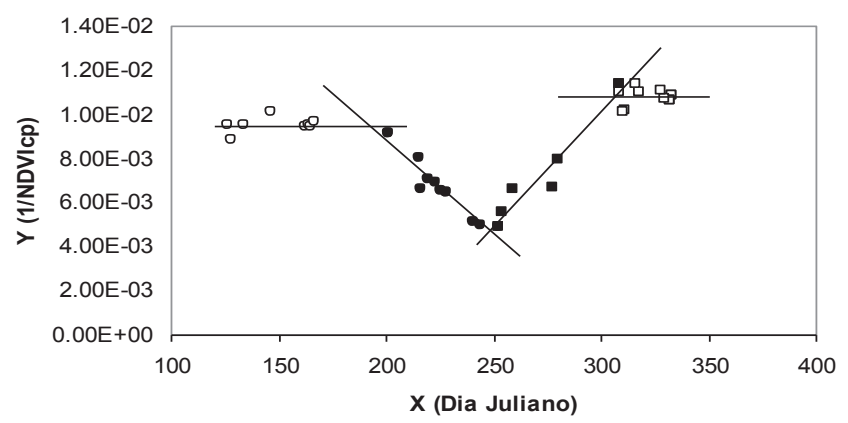

Figura 6. Ajuste del algoritmo de reconocimiento de patrones a la curva de crecimiento de un píxel individual. EI NDVIcp se multiplicó por 1000 . 
paramétrico satelital. La valoración del riesgo utiliza los umbrales de pago asociados a eventos catastróficos entre el año $\mathrm{k}$ de cobertura del seguro y el anterior k-1 (Paz et al., 2018):

$$
\text { Si } I S_{k} \leq\left(I S_{k-1}\right) F A C A \text {, entonces pago seguro }
$$

donde FACA (0.64) es un factor de ajuste del coeficiente de agostadero (capacidad de carga) que refleja el evento catastrófico (condición corporal de ganado sin grasa). vi) Para estimar la capacidad de carga de los municipios (monto máximo de la cobertura del seguro) con pastizales naturales/inducidos y matorrales (INEGI, 1997), se consideraron las estimaciones de coeficientes de agostadero de COTECOCA (1967) que se encuentran en las memorias estatales de México (décadas de 1960 y 1970, principalmente). Dado que los datos de capacidad de carga (inverso del coeficiente de agostadero) de COTECOCA no están actualizados, se aproximaron a las condiciones actuales de vegetación, al usar como mapa base la Serie III de uso del suelo del INEGI, año base 2002 (INEGI, 2002). Un proceso similar se siguió para la Serie IV con año base 2007.

\section{Campañas Experimentales de Validación}

La validación del desarrollo teórico del seguro satelital se llevó a cabo en una campaña inicial, piloto, en el año 2005, en los municipios de Naquimipa, estado de Chihuahua e Hidalgo, estado de Durango (Figura 7). Se hicieron mediciones en 23 pastizales naturales de cada municipio, con visitas durante la temporada de crecimiento vegetativo y senescencia. El arreglo experimental, equipo de medición de la campaña, así como los resultados de la validación del modelo de geometría sol-sensor, se documentan en Bolaños et al. (2007).

Durante la campaña de muestreo, en los sitios de control se hicieron mediciones pareadas de radiometría (radiómetro de 5 bandas espectrales), cobertura vegetal a partir de fotografías digitales y biomasa aérea seca; adicionalmente, en los sitios de muestreo se midió radiometría y cobertura vegetal.

En el 2007 se realizó una campaña intensiva en diferentes estados de la República Mexicana (Durango, Coahuila, Jalisco, Zacatecas, Querétaro y Estado de México), Figura 8. Los protocolos experimentales

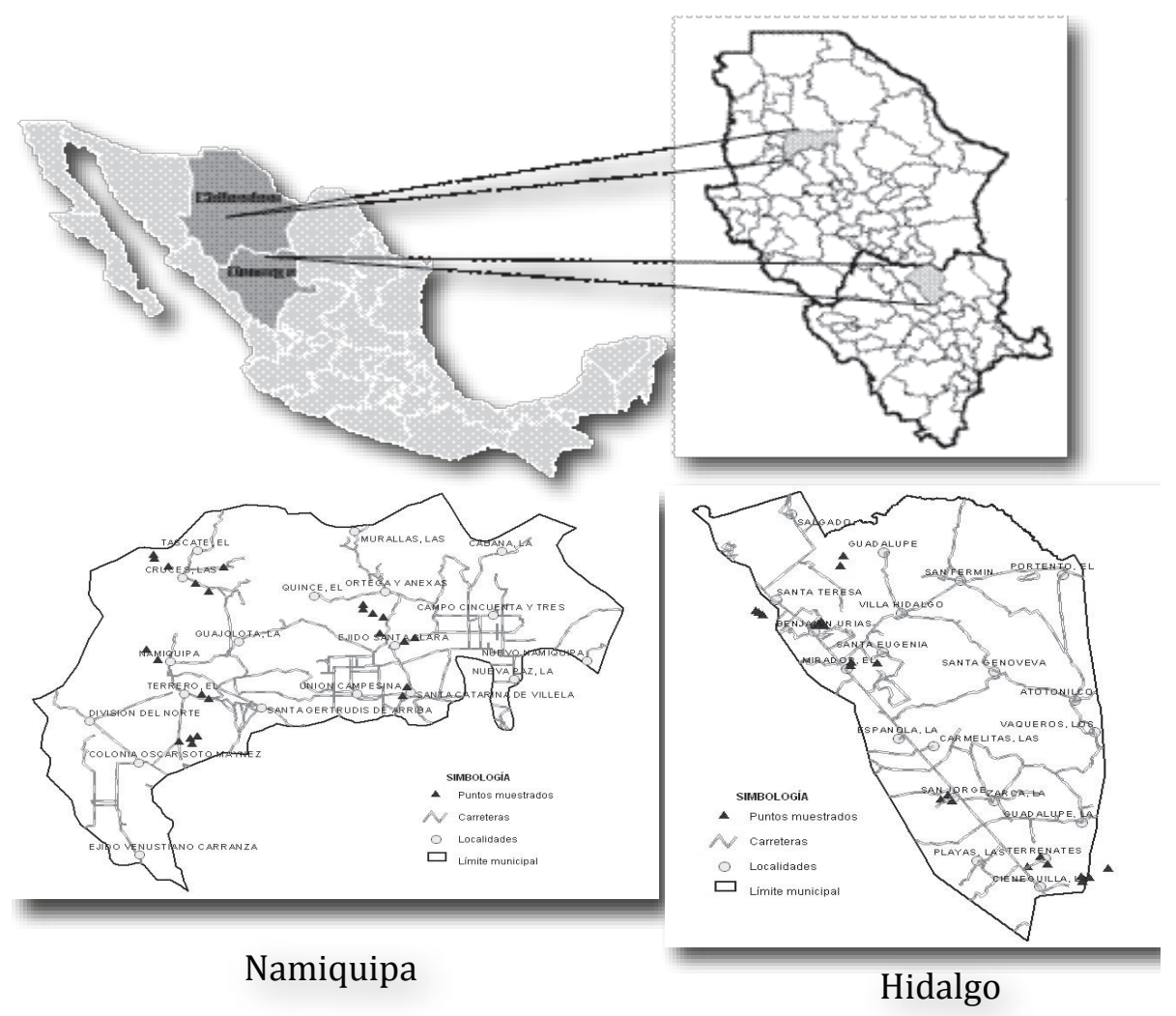

Figura 7. Fase inicial, piloto, de la validación del seguro satelital y sus elementos. 


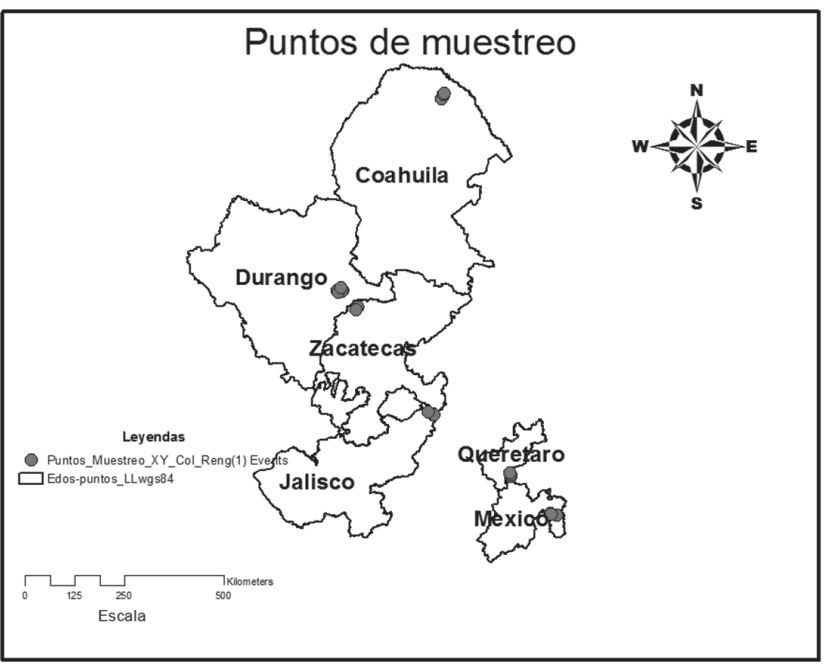

Figura 8. Localización de los sitios de muestreo durante la campaña del 2007.

se ampliaron para obtener un mayor número de mediciones pareadas de radiometría, cobertura aérea y biomasa, en pastizales naturales e inducidos (sensu INEGI). Se incluyeron mediciones hechas con el uso de un helicóptero de control remoto instrumentado (Bolaños et al., 2010). Como prueba de concepto se efectuaron mediciones de mayor intensidad espacial
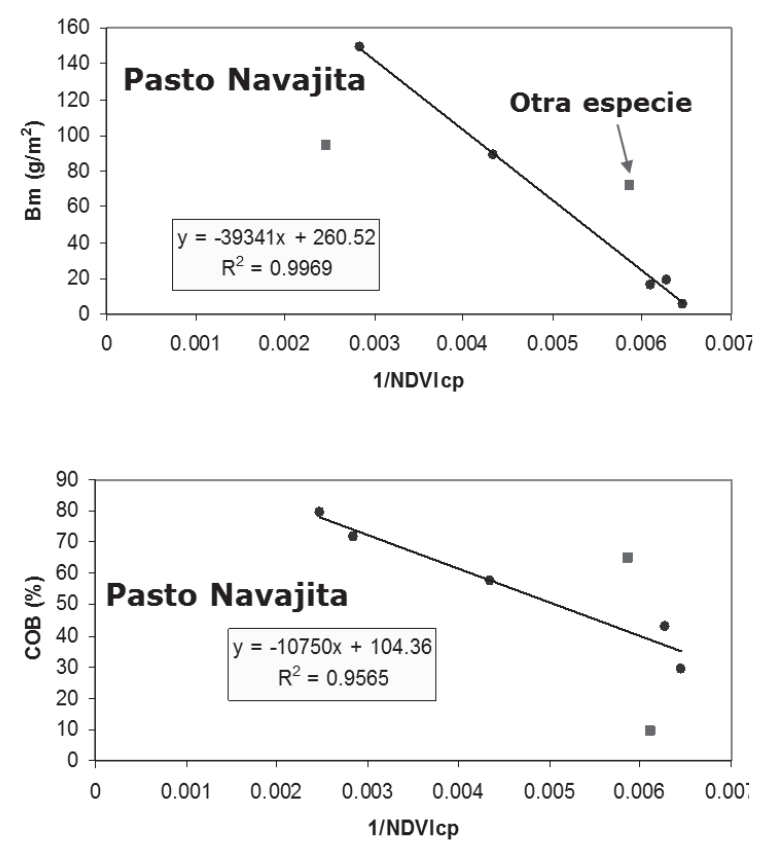

en el rancho Atotonilco, en el estado de Durango, para analizar el desarrollo de seguros paramétricos a escala de predios o ranchos, además de analizar el impacto de estos productos (estimaciones de capacidad de carga y curvas de crecimiento a escala de pixeles) en la toma de decisiones de los productores pecuarios.

Los modelos que se utilizaron para el ajuste de los datos experimentales fueron:

$$
\begin{aligned}
& B m=u I V C P \\
& B m=v+w C O B
\end{aligned}
$$

donde: $\mathrm{u}, \mathrm{v}$ y w son constantes empíricas representativas de los diferentes tipos de pastizales que se analizaron.

\section{RESULTADOS Y DISCUSIÓN}

\section{Experiencia Operacional de la Implementación del Seguro}

El esquema experimental consistió en obtener, para los sitios control, las relaciones IV (1/NDVIcp) y biomasa, así como IV y cobertura aérea (COB) y extenderlas a otros sitios mediante una estimación indirecta de la biomasa aérea total (sólo pastos). La Figura 9 muestra algunos de los resultados.
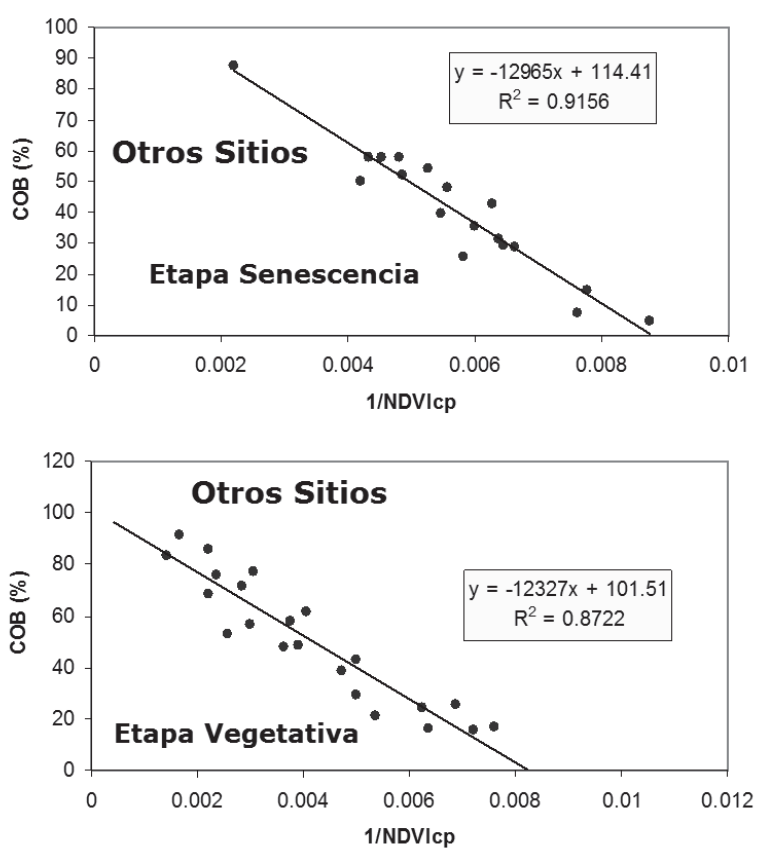

Figura 9. Relación (1/NDVIcp)-Bm y COB para un sitio de control (pasto navajita; Bouteloa spp.) y (1/NDVIcp)-COB para sitios no control, etapa vegetativa y de senescencia. $\mathrm{Bm}=$ biomasa; $\mathrm{COB}=$ cobertura aérea. 
Las estimaciones satelitales, en relación con las mediciones de campo, se revisaron con conocimiento de los errores residuales del monitoreo satelital y los efectos de escala, mediante el concepto de equivalencia ambiental (Paz et al., 2009), que supone que las relaciones entre los parámetros de las etapas de las curvas de crecimiento de los pastizales, ecuación (2), definen patrones específicos. Al comparar ambas fuentes de información, el efecto atmosférico (radianza de trayectoria, constante aditiva de los aerosoles) y de escala (constante multiplicativa) fueron los de mayor impacto, por lo que las pendientes en los espacios paramétricos son más o menos similares, pero se desfasan en el origen (constantes aditivas). La Figura 10 muestra los espacios paramétricos entre las mediciones de campo y las satelitales, con los patrones descritos.

La Figura 11 muestra los resultados de la campaña de 2007 en distintos sitios de muestreo para diferentes estados. Las relaciones IVCP-Bm fresca y COB$\mathrm{Bm}$ fresca, se estandarizaron para poder compararlas entre sí. Generalmente, en cada sitio de muestreo hay una relación única de las ecuaciones (11), constantes empíricas locales, las cuales se usaron para realizar los gráficos de la Figura 11.

En el rancho Atotonilco, los muestreos se distribuyeron espacialmente para obtener relaciones entre la $\mathrm{COB}$ y la $\mathrm{Bm}$ fresca (entre otras), que representaran ambientes diferentes, de tal forma que pudieran estandarizarse para validar el enfoque y calibrar la información terrestre y satelital (Figura 12).

Ambos municipios

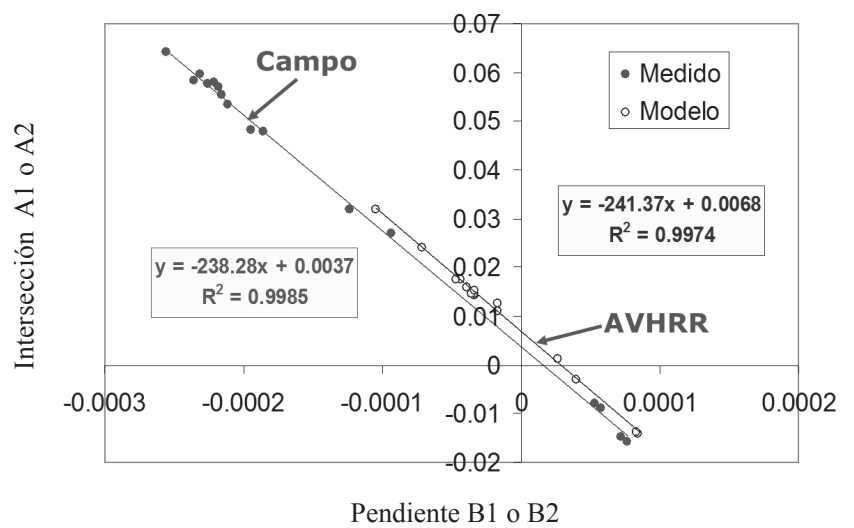

Figura 10. Espacios paramétricos de las etapas vegetativa y de senescencia de los pastizales, con mediciones de campo y satelitales.
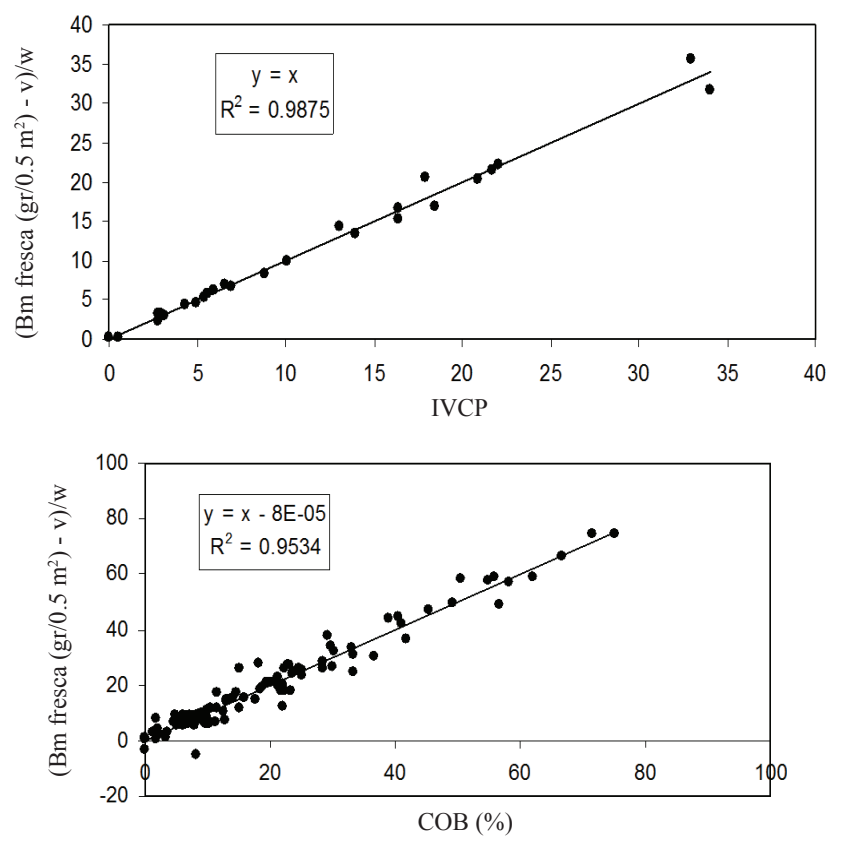

Figura 11. Relaciones estandarizadas ente el IVCP y la Bm fresca y la $\mathrm{COB}$ y la $\mathrm{Bm}$ fresca de diferentes sitios de muestreo de la campaña del 2007. $\mathrm{Bm}=$ biomasa; $\mathrm{COB}=$ cobertura aérea.

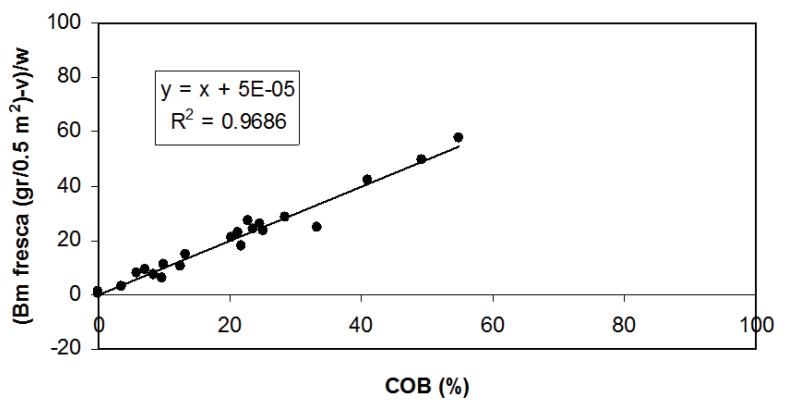

Figura 12. Relación entre la $\mathrm{COB}$ y la $\mathrm{Bm}$ fresca en diferentes sitios de muestreo del rancho Atotonilco, Durango. $\mathrm{Bm}=$ biomasa $\mathbf{C O B}=$ cobertura aérea.

Del análisis de las variables biofísicas y radiométricas (IV) se obtuvieron relaciones consistentes y congruentes con el marco teórico, con ajustes empíricos adecuados.

La Figura 13 muestra el espacio paramétrico de las etapas vegetativa (creciente) y de senescencia (decreciente), de la curva temporal de la biomasa en los sitios de la campaña experimental (con información suficiente para caracterizarla), el cual concuerda con el concepto de equivalencia ambiental que se usó para validar patrones genéricos en sitios de muestreo con diferentes ambientes (diferencias en el inicio del ciclo de crecimiento) y niveles de estrés (Paz et al., 2009). 


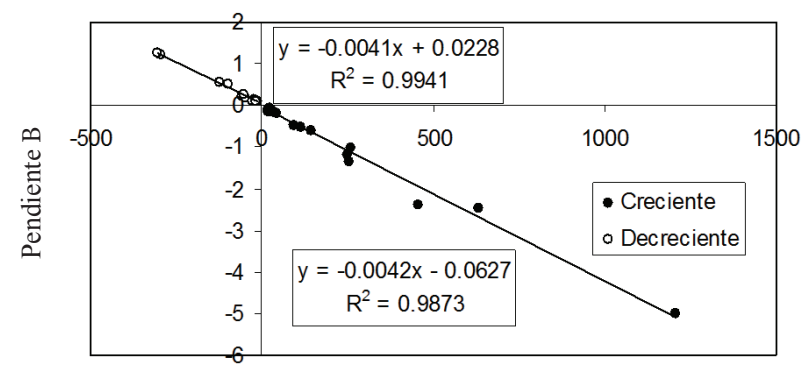

Intersección A

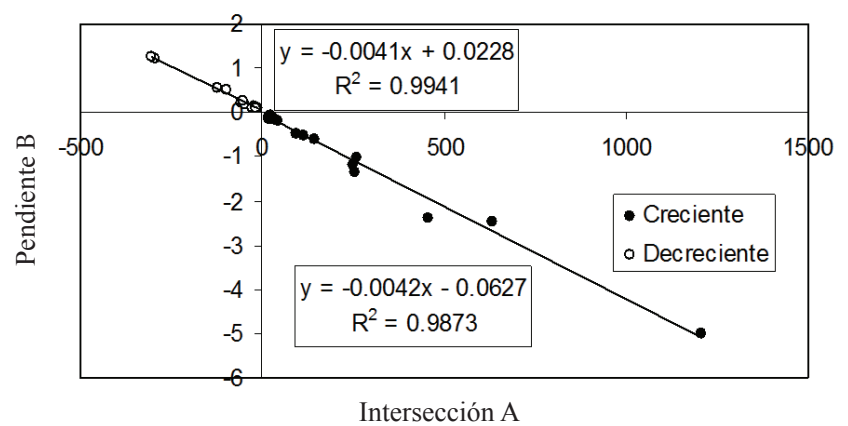

Figura 13. Patrones entre los parámetros de la evolución temporal de la biomasa evaluada en campo, para la etapa vegetativa (creciente) y de senescencia (decreciente).

Con la finalidad de verificar visualmente $\mathrm{y}$, con fotografías digitales, los resultados de los productos satelitales, se realizaron campañas nacionales durante otros años de operación del seguro paramétrico satelital, de tal forma que se establecieron validaciones de las observaciones con relación a las estimaciones por sensores remotos.

\section{Operación del Seguro Paramétrico en México}

En el Cuadro 1 se muestran las estadísticas de superficie, uso del suelo y cobertura de unidades animal (UA) durante el periodo 2005 a 2010. Por otra parte, y a manera de síntesis, la Figura 14 muestra la evolución de la operación del seguro ganadero paramétrico satelital en México, desde las pruebas sin validez jurídica del 2005 y 2006, hasta su implementación operacional a escala de municipios a partir del 2007.

Cuadro 1. Estadísticas de la operación del seguro ganadero paramétrico satelital.

\begin{tabular}{llcc}
\hline \multirow{2}{*}{ Año } & Uso del suelo & Superficie & $\begin{array}{c}\text { Unidades } \\
\text { animal }\end{array}$ \\
\cline { 3 - 4 } & & Millones ha ${ }^{-1}$ & Millones \\
\hline $2005^{*}$ & Pastizal natural e inducido & 0.44 & 0.03 \\
$2006^{* *}$ & Pastizal natural e inducido & 8.00 & 0.45 \\
2007 & Pastizal natural e inducido & 13.00 & 0.90 \\
2008 & $\begin{array}{l}\text { Pastizal natural e inducido } \\
\text { y matorral }\end{array}$ & 58.00 & 2.70 \\
2009 & $\begin{array}{l}\text { Pastizal natural e inducido } \\
\text { y matorral }\end{array}$ & 54.00 & 3.05 \\
& $\begin{array}{l}\text { Pastizal natural e inducido } \\
\text { y matorral }\end{array}$ & 57.00 & 3.15 \\
\hline Hidalgo y Namiquipa (prueba piloto).
\end{tabular}

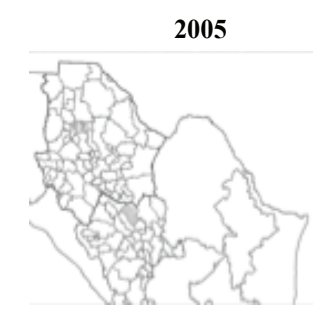

2007
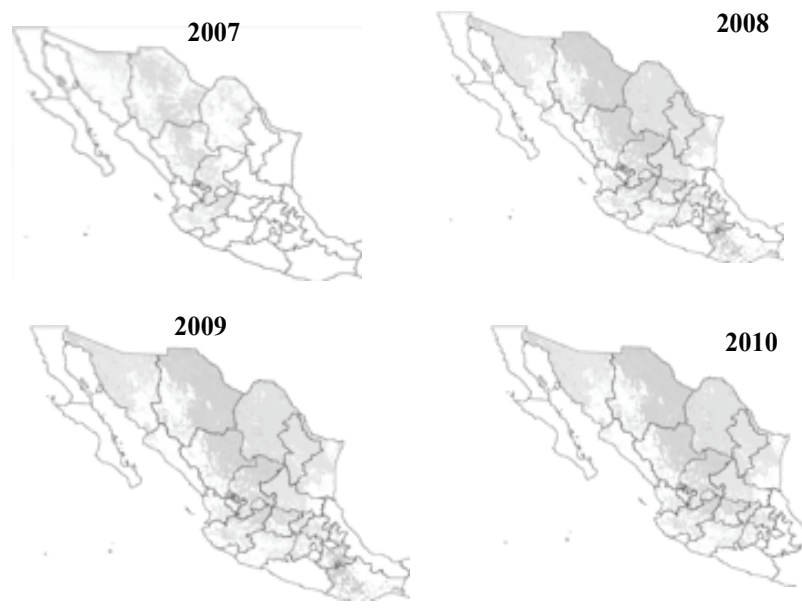

2008

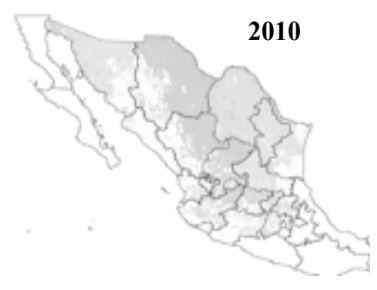

Figura 14. Evolución de la cobertura de superficie del seguro ganadero paramétrico satelital, del 2005 al 2010. 


\section{CONCLUSIONES}

- Los resultados de la experiencia mexicana en la implementación operacional de seguros ganaderos paramétricos satelitales (del tipo catastrófico), operada por AGROASEMEX, S.A. (paraestatal a cargo de los seguros en México), mostro resultados favorables en el uso de la tecnología satelital, acoplada a conocimiento científico, y fue el primer caso de este esquema en América Latina (y otros continentes), posicionando a México a la vanguardia de desarrollos de seguros paramétricos operacionales.

- Si el seguro satelital considera un ciclo completo (entre $\mathrm{X}_{\mathrm{i}} \mathrm{y}_{\mathrm{f}}$ ) el índice de seguro sólo contempla el crecimiento de la vegetación entre las dos fechas, esquema que, aún cuando reduce los riesgos de base que conlleva el uso de periodos cortos del seguro, como la cercanía de las estimaciones satelitales con la realidad, mantiene el problema de los ciclos de crecimiento desfasados, es decir, de inicios tempranos o tardíos, restricción que es inherente a considerar una fecha inicial y una final de vigencia del seguro paramétrico.

- Los nuevos desarrollos que pueden ser planteados en función de la experiencia operacional adquirida consisten en: (a) Uso de índices de vegetación más robustos y que consideren etapas más allá de la vegetativa; (b) consideración de los tiempos de colocación de los seguros con relación a los inicios tempranos o tardíos del ciclo de crecimiento de la vegetación; (c) uso de sensores de mayor resolución espacial y estabilidad (p. ej.: MODIS); y, (d) desarrollo de enfoques orientados a productores individuales.

- Finalmente, es importante enfatizar que los productos satelitales discutidos están basados en conocimiento científico de la transferencia de radiación en el sistema atmosfera-vegetación-suelo, sujeto a los requerimientos para reducir o eliminar el riesgo de base, por lo que el uso de productos con bases empíricas (otros indicies de vegetación y algoritmos) no responden a esta condición, incrementando su riesgo de base y con probabilidades altas de que cuando se presente un siniestro en los pastizales y matorrales de México su operación no refleje esta situación, dejando expuestos a los estados y productores.

\section{LITERATURA CITADA}

AGROASEMEX, S.A. 2005. Administración de riesgos agropecuarios. Manuales para el fortalecimiento de los fondos de aseguramiento agropecuario. AGROASEMEX, S.A. Querétaro, México.

AGROASEMEX, S.A. 2006a. La experiencia mexicana en el desarrollo y operación de seguros paramétricos orientados a la agricultura. AGROASEMEX, S.A. Querétaro, México. ISBN 968-9098-00-4.

Arias, D. and K. Covarrubias. 2006. Agricultural insurance in Mesoamerica: An opportunity to deepen rural financial markets. Inter American Development Bank. Economic and Sector Study Series. RE2-06-006. Washington, DC, USA.

Arnold, J. G., M. A. Weltz, E. E. Alberts, and D. C. Flanagan. 1995. Plant growth component. Chapter 8. pp. 8.1-8.41. In: D. C. Flanagan and M. A. Nearing (eds.). USDA-Water Erosion Prediction Project (WEPP). Hillslope profile and watershed model documentation. NSERL Report No. 10. USDA-ARS National Soil Erosion Research Laboratory. West Lafayette, IN, USA.

Bolaños, M., F. Paz, E. Palacios, E. Mejía y A. Huete. 2007. Modelación de los efectos de la geometría sol-sensor en la reflectancia de la vegetación. Agrociencia 41: 527-537.

Bolaños, M., F. Paz, E. Palacios y J. Navarro. 2010. Medición de reflectancias espectrales en especies forestales utilizando un helicóptero de radio control. Terra Latinoamericana 28: 185-189.

Chehbouni, A., Y. H. Kerr, J. Qi, A. R. Huete, and S. Sorooshian. 1994. Toward the development of a multidirectional vegetation index. Water Resour. Res. 30: 1281-1286. doi: 10.1029/93WR03063.

COTECOCA (Comisión Técnico Consultiva de Coeficientes de Agostadero). 1967. Metodología para determinar tipos vegetativos, sitios y productividad de sitios. Publicación No. 8. México, D. F.

Emmick, D. L. 2000. Prescribed grazing and feeding management for lactating dairy cows. New York State Grazing Lands Conservation Initiative in Cooperation with USDA-Natural Resources Conservation Service. Syracuse, NY, USA.

Goodrum, G., K. B. Kidwell, and W. Winston. 2001. NOAA KLM users guide. NOAA-NESDIS-NCDC, Suitland, MD. http://www2.ncdc.noaa.gov/docs/klm/index.htm (Consulta: septiembre 13, 2009).

Goudriaan, J. and H. H. van Laar. 1994. Modelling potential crop growth processes. Text book with exercises. Current Issues in Production Ecology. Springer. Netherlands.

Holechek, J. L., R. D. Pieper, and C. H. Herbel. 1989. Range management, principles and practices. Prentice Hall. Englewood Cliffs, NJ, USA. ISBN-13: 978-0135014165.

Huete, A. R., R. D Jackson, and D. F. Post. 1985. Spectral response of a plant canopy with different soil backgrounds. Remote Sens. Environ. 17: 35-53. doi: 10.1016/0034-4257(85)90111-7.

Huete, A. R., G. Hua, J. Qi, and A. Chehbouni. 1992. Normalization of multidirectional red and NIR reflectances with the SAVI. Remote Sens. Environ. 41: 143-154. doi: 10.1016/00344257(92)90074-T. 
Kidwell, K. B. 1998. NOAA polar orbiter data user's guide (TIROS-N, NOAA-6, -7, -8, -9, -10, -11, -12, -13, and -14). NOAA-NESDIS-NCDC, Suitland, MD, USA. http://www2. ncdc.noaa.gov/docs/podug/index.htm. (Consulta: septiembre 13, 2009).

INEGI (Instituto Nacional de Estadística y Geografía). 1997. Diccionario de datos de uso del suelo y vegetación, escala 1:250 000 (vectorial), Base de Datos Geográficos. Instituto Nacional de Estadística, Geografía e Informática. Aguascalientes, México.

INEGI (Instituto Nacional de Estadística y Geografía). 2002. Carta de uso actual del suelo y vegetación serie III. Escala 1:250 000 (vectorial). Base de datos geográficos. Instituto Nacional de Estadística y Geografía. Aguascalientes, México.

Paz, F., E. Palacios, M. Bolaños, A. Cano, A. Zarco, F. Pascual, L. A. Palacios, and M. Martínez. 2006. Design of a country scale livestock insurance in grasslands using AVHRR sensor. pp. 683-685. In: J. A. Sobrino (ed.). Second recent advances in quantitative remote sensing. Universitat de Valencia. Valencia, Spain

Paz, F., E. Palacios, M. Bolaños, L. A. Palacios, M. Martínez, E. Mejía y A. Huete. 2007. Diseño de un índice espectral de la vegetación: NDVIcp. Agrociencia 41: 539-554.

Paz, F., M. Bolaños, E. Palacios, L. A. Palacios, M. Martínez y A. Huete. 2008. Optimización del índice espectral de la vegetación NDVIcp. Agrociencia 42: 925-937.

Paz, F., M. Odi, A. Cano, M. A. Bolaños y A. Zarco. 2009. Equivalencia ambiental en la productividad de la vegetación. Agrociencia 43: 635-648.
Paz, F., Bolaños, M., L. A. Palacios, F. Pascual, E. Medrano y F. Ibarra. 2011. Desarrollo de curvas espectrales del crecimiento anual de la vegetación, usando sensores remotos. Rev. Mex. Cienc. Pecu. 2: 445-464.

Paz, F., M. Bolaños, F. Pascual, J. Escamilla, M. Cuesta y J. I. Zúñiga. 2018. Experiencia mexicana en el diseño de seguros ganaderos paramétricos satelitales de tipo catastrófico: bases conceptuales y teóricas. Terra Latinoamericana 36: 135-144. doi: 10.28940/terra.v36i2.227.

Qi, J., A. R. Huete, F. Cabot, and A. Chehbouni. 1994. Bidirectional properties and utilization of high-resolution spectra from a semiarid watershed. Water Resour. Res. 30: 1271-1279. doi: 10.1029/93WR03058.

Stockle, C. O., M. Donatelli, and R. Nelson. 2003. CropSyst, a cropping systems simulation model. Eur. J. Agron. 18: 289-307.

Stowe, L. L., P. A. Davis, and E. P. McClain. 1999. Scientific basis and initial evaluation of the CLAVR-1 global clear/cloud classification algorithm for the advanced very high resolution radiometer. J. Atmos. Oceanic Technol. 16: 656-681. doi: 10.1175/1520-0426(1999)016<0656:SBAIEO >2.0.CO;2.

Tucker, C. J. 1979. Red and photographic infrared linear combinations for monitoring vegetation. Remote Sens. Environ. 8: 127-150.

USDA (United States Department of Agriculture). 1999. Technical support for the NIRS/NUTBAL nutritional management system. USDA-NRCS-GLTI. Fort Worth, TX, USA.

Verstraete, M. M. and B. Pinty. 1996. Designing optical spectral indexes for remote sensing applications. IEEE Trans. Geosci. Remote Sens. 34: 1254-1265. doi: 10.1109/36.536541. 